\title{
Lung density analysis using quantitative computed tomography in children with pectus excavatum
}

\author{
Fatma C. Sarioglu ${ }^{1 A, B, C, C, E, E, F}$, Naciye S. Gezer ${ }^{1 A, D, E}$, Huseyin Odaman ${ }^{1 A, B, D, F}$, Orkun Sarioglu ${ }^{2 A, B, C, E}$, \\ Oktay Ulusoy ${ }^{3 A, B, D, F}$, Oguz Ates ${ }^{3 A, B, D}$, Handan Guleryuz ${ }^{1 A, B, D, F}$ \\ 'Department of Radiology, Dokuz Eylul University School of Medicine, Izmir, Turkey \\ 2Department of Radiology, Izmir Democracy University School of Medicine, Izmir, Turkey \\ ${ }^{3}$ Department of Pediatric Surgery, Dokuz Eylul University School of Medicine, Izmir, Turkey
}

\section{Abstract}

Purpose: To evaluate the mean lung density in children with pectus excavatum (PE) and to assess the correlation between the cardiac rotation angle, Haller index, pulmonary function test, and lung density.

Material and methods: This retrospective study included 33 children with PE and 31 healthy controls. The densities of lung parenchyma were evaluated by quantitative computed tomography (CT). Three lung levels were determined: T4 vertebra level, T10 vertebra level, and the level of the measurement of the cardiac rotation angle. The cardiac rotation angle and the Haller index were calculated. All measurements were done by 2 radiologists, independently. Student's $t$-test or the Mann-Whitney $U$ test, intraclass correlation coefficients, Pearson or Spearman's rank correlation coefficient, and Kruskal-Wallis test were used for statistical analysis. A p-value less than 0.05 was considered as statistically significant.

Results: All the lung levels in the PE group had lower mean densities than healthy children, with statistical significance in the right lung at the T10 vertebra level $(-818.60 \pm 33.49 \mathrm{HU},-798.45 \pm 40.24 \mathrm{HU} ; p=0.028)$. There was a correlation between the cardiac rotation angle and the Haller index $(\rho=0.593 ; p<0.001)$. There were no correlations between mean lung density and cardiac rotation angle, Haller index, and pulmonary function tests.

Conclusions: The lower mean lung densities were found in PE, especially in the right lower lobe. The parenchymal aeration should be considered independently from the severity of PE.

Key words: Haller index, lung density, pectus excavatum, paediatric, quantitative computed tomography.

Pectus excavatum (PE) is the most common congenital chest wall deformity with an incidence of 1/300-400 live births [1]. Displacement of the sternum and costosternal joints to posterior is the main occasion in PE. The condition often results in cardiopulmonary symptoms and psychological problems [2-5]. The most commonly associated anomalies are scoliosis, Marfan syndrome, congenital heart disease (most commonly mitral valve prolapse), rickets, Poland's syndrome, and osteogenesis imperfecta [6].
Chest computed tomography (CT) is a key component to assess the mediastinal shift, cardiac compression, and the lung parenchymal abnormalities such as atelectasis or tracheobronchial compression in patients with PE. Indeed, subjective evaluations of the abnormal inflation that may be related to mediastinal shift and sternal depression on thorax CT scans have been found in PE patients [5,7]. However, the visual analysis may cause substantial variability in the subjective assessment among the readers. Quantitative CT analysis can reveal the exact

Correspondence address:

Fatma C. Sarioglu, Department of Radiology, Dokuz Eylul University School of Medicine, Izmir, Turkey, e-mail: drcerenunal@gmail.com

Authors' contribution:

A Study design · B Data collection · C Statistical analysis · D Data interpretation · E Manuscript preparation · F Literature search · G Funds collection 
pixel densities of the entire lung or a specific area. Moreover, quantitative CT detects and calculates the percentage of abnormal parenchymal densities that would be extremely difficult to distinguish by visual analysis [8].

There have been several articles assessing lung parenchymal density in many acquired pulmonary diseases such as chronic obstructive pulmonary disease and interstitial lung disease, by using a sensitive density measurement method: quantitative CT [9-12]. Nonetheless, there is a lack of studies conducted by quantitative CT in congenital thoracic wall deformities even though the evaluation of parenchymal abnormalities in PE has recently become an area of interest [5].

We hypothesized that parenchymal hyperinflation may occur in patients with PE, especially in lower lung lobes. Thus, the purpose of this study is to evaluate the mean lung parenchymal density in children with PE comparing with healthy controls. The secondary goal is to assess the correlation between the cardiac rotation angle, Haller index, pulmonary function test, and parenchymal density in PE.

\section{Material and methods}

\section{Study population}

After receiving approval from Dokuz Eylul University School of Medicine institutional review board (2019/ 27-45), chest CT images of children with any thoracic wall deformity, who were admitted to the paediatric surgery department between January 2008 and March 2019, were retrospectively evaluated. The inclusion criteria were children between 0 and 18 years old with $\mathrm{PE}$, and those with thorax CT imaging before PE repair surgery $(n=107)$. Exclusion criteria were children who had contrast-enhanced CT images $(n=25)$, alveolar or interstitial lung disease $(n=3)$, chemotherapy or radiotherapy history $(n=1)$, and images with motion artifacts $(n=31)$. Due to the extremely sensitive nature of quantitative CT analysis, children who had minimal artifacts in their chest CT were excluded to obtain accurate results. Normal mean values of the Haller index are described as less than 2.5 [13]. Patients with Haller index less than 2.5 were not accepted in the PE group $(n=14)$. After the inclusion and exclusion criteria, 33 patients were enrolled in this study.

As a control group, we scanned the chest CTs of paediatric patients who had thorax CT scanning between January 2008 and March 2019. Among these patients, only children with no abnormal findings on the CT scans were selected for the control group. The same exclusion criteria were used for the control group. Children with a Haller index higher than 2.5 who were detected incidentally were also excluded. Finally, 31 children were included in the control group. The parents of the children provided informed consent for imaging.

\section{Computed tomography protocols}

At our institution, CT scanning is routinely performed in full expiration for children. Verbal instructions and deep breathing exercises are performed before CT scanning. General anaesthesia or sedation is not performed while CT scanning.

CT images were acquired using a multi-detector CT scanner (Toshiba Aquilion 64; Toshiba Medical Systems, Otawara, Japan). Acquisitions were performed with no contrast injection and with the following parameters: $100 \mathrm{kV}$ tube voltage, $50 \mathrm{mAs}$ tube current, $3 \mathrm{~mm}$ slice thickness, and $0.6 \mathrm{~mm}$ collimation. CT dose index ranged from 0.67 to $7.5 \mathrm{mGy}$. Images were displayed with parenchymal 1600 Hounsfield units (HU) window width and $-500 \mathrm{HU}$ window level.

\section{Image interpretation}

\section{Quantitative lung density analysis}

Quantitative lung density analysis was performed using a semi-automated technique with the software package (Myrian 2.4.2., Intrasense, Montpellier, France). Three lung levels were determined for quantitative analysis of lung parenchymal density to observe the following: T4 vertebra spinous process level for upper lobes, T10 vertebra spinous process level for lower lobes, and the level at which the cardiac rotation angle was measured. The last level was determined to assess the effect of cardiac rotation on the lung parenchyma. All measurements were done for both lungs. Hereafter, we used "T4R" and "T4L" for the right lung and left lung at the T4 vertebra level, "T10R" and "T10L" for the right lung and left lung at the T10 vertebra level, and "CRAR" and "CRAL" for the right lung and left lung at the cardiac rotation angle level. The T9 level was used when the lung parenchyma entering the slice at the T10 level was less than $75 \%$ of the total slice. The lung parenchyma was manually drawn on each slice to be the closest distance to the pleura (Figure 1). While drawing the medial border at the CRAR and CRAL levels, motion-induced densities were not included in the region of interest (ROI). Automated measurement of lung parenchymal density was obtained, and the parenchymal density distribution between -650 and -1050 was recorded as percentages.

\section{Cardiac rotation angle}

The cardiac rotation angle was accepted as the angle between the cardiac apex and the line between the anterior vertebral corpus and the spinous process (Figure 2).

\section{Haller index}

The Haller index was calculated on each participant utilizing the formula proposed by Haller et al. [13]: maximal trans- 

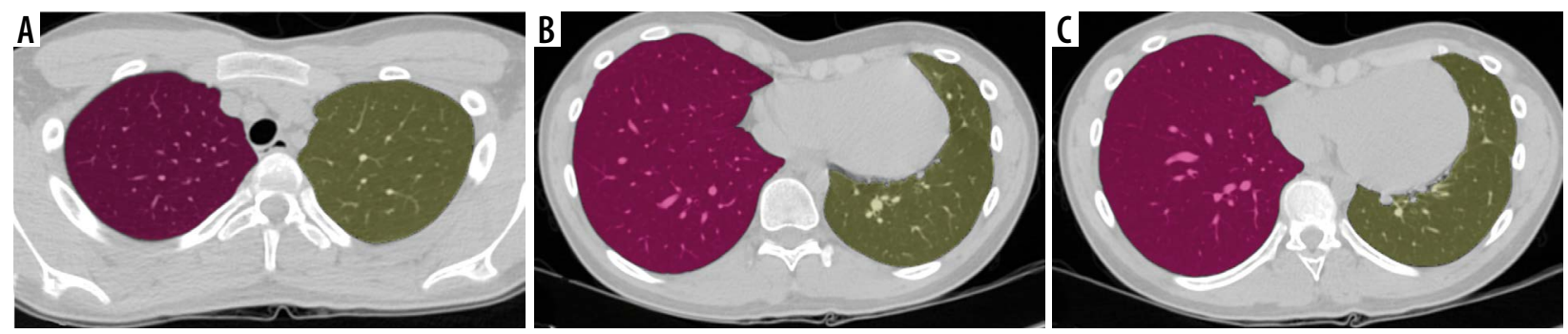

Figure 1. The drawing method used for analysis of the selected lung levels. A) T4 vertebra spinous process level. B) T10 vertebra spinous process level. C) The level of the measurement of cardiac rotation angle
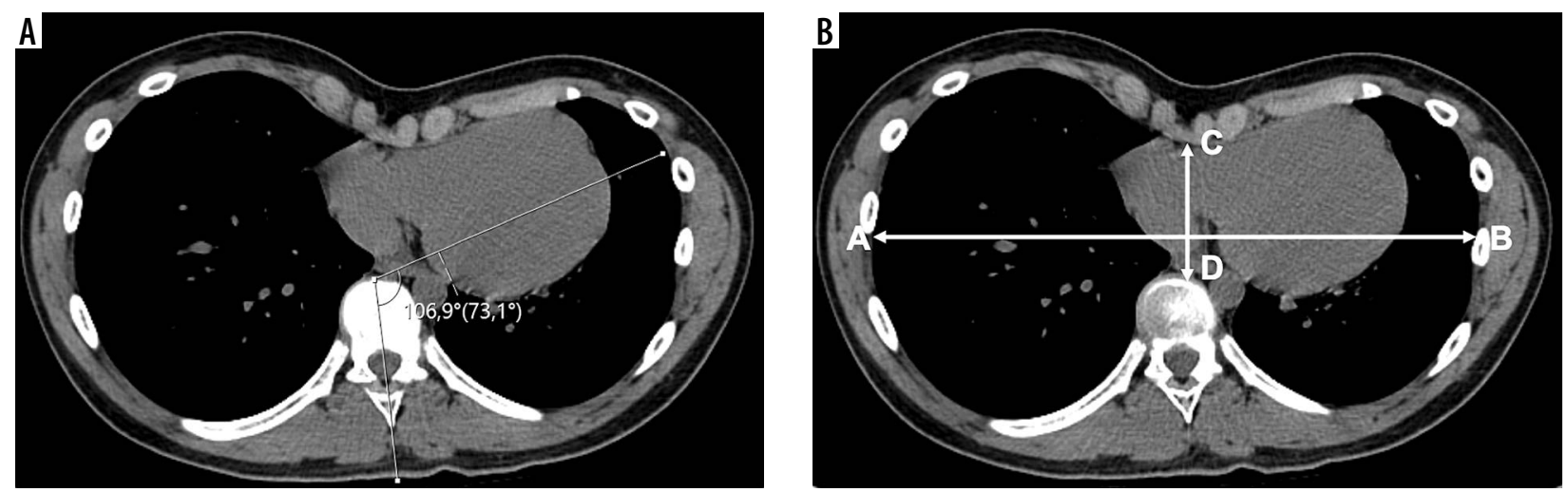

Figure 2. A) The method of the calculation of the cardiac rotation angle. B) The method of the calculation of the Haller index; $A B / C D$

verse diameter of the chest divided by the anterior-posterior distance on the axial slice, which demonstrates the smallest distance between the anterior surface of the vertebral body and the posterior surface of the sternum (Figure 2). The PE was severe if the Haller index was greater than $3.2[14,15]$.

A radiologist with 8 years of experience and 3 years of experience in paediatric radiology, and a radiology resident with 3 years of experience performed the measurements individually. All measurements were performed twice by each radiologist.

\section{Pulmonary function test}

Spirometry (ZAN 510, Oberthulba, Germany) was performed according to the American Thoracic Society guidelines [16]. Spirometry provided the forced vital capacity (FVC), forced expiratory volume in $1 \mathrm{~s}\left(\mathrm{FEV}_{1}\right)$, and the $\mathrm{FEV}_{1} / \mathrm{FVC}$ ratio. The FVC and $\mathrm{FEV}_{1}$ were reported as the percentage predicted according to age, gender, and height. Normal spirometry findings were defined as FVC, $\mathrm{FEV}_{1}$, and $\mathrm{FEV}_{1} / \mathrm{FVC}$ ratio $>80 \%$ of the predicted value according to age, gender, and height. Obstructive lung function was defined as $\mathrm{FEV}_{1}<80 \%$ of predicted value and $\mathrm{FEV}_{1} / \mathrm{FVC}<80 \%$. Restrictive lung function was described as a reduced $\mathrm{FVC}<80 \%$ of predicted value, with a preserved $\mathrm{FEV}_{1} / \mathrm{FVC}$ ratio $>80 \%$. The mean time between pulmonary function test administration and chest CT scanning was 7 days \pm 11 (range, 0-50 days).

\section{Statistical analysis}

Statistical analyses were made using IBM SPSS ver. 22.0 (IBM Corp., Armonk, NY, USA). Categorical variables were summarized with frequency counts and percentages, and the continuous features were summarized with means and standard deviations. The distribution of the variables was performed using Kolmogorov-Smirnov and Shapiro-Wilk tests. Continuous variables were compared by using the Student's $t$-test or the Mann-Whitney $U$ test. Interobserver agreements of the cardiac rotation angle, Haller index, and mean lung densities in each level were tested for intraclass correlation coefficients. The correlation between mean lung densities of each level and Haller index, cardiac rotation angle, and pulmonary function tests were measured with the Pearson or Spearman's rank correlation coefficient in the PE group. Correlation was considered slight when the correlation coefficient was $<0.2$, fair when 0.2-0.4, moderate when 0.4-0.6, substantial when 0.6-0.8, and excellent when $>0.8$. The Kruskal-Wallis test was used to compare the mean values of the patients according to the lung function tests. A $p$-value less than 0.05 was considered as statistically significant.

\section{Results}

In this study, 33 patients (girls/boys, 9/24; mean age, 14.42 years \pm 2.25 ; age range, 10 to 18 years) and 31 healthy controls (girl/boy, 6/25; mean age, 14.61 years \pm 2.09 ; age range, 10 to 18 years) were included. There were no significant differences in age and sex between the groups $(p=0.73 ; p=1.0)$. The number of patients with Haller index $<3.2$ was $13(39.3 \%)$, while $>3.2$ was 20 (60.6\%). There were no patients who had a syndrome associated with PE. One patient had mild scoliosis. Demographic features and patients' characteristics are shown in Table 1. 
Echocardiography revealed mitral valve prolapse in one patient with a Haller index of 3.05.

Excellent interobserver agreements were noted for quantitative analysis of the lungs (mean lung density values in each level, measurements of the Haller index and the cardiac rotation angle). The mean values of the $2 \mathrm{ob}$ servers for each parameter were used in the final analyses because of the excellent agreement. The lowest mean lung density was found at the T4L level in the control group (mean, $-801.23 \pm 40.67 \mathrm{HU}$ ), T10R level in the PE (mean, $-818.60 \pm 33.49 \mathrm{HU}$ ). In comparison of mean lung densities of each level between two groups, significantly lower values were found at the T10R lung level in the PE group $(-818.60 \pm 33.49 \mathrm{HU},-798.45 \pm 40.24 \mathrm{HU}$; $p=0.028$ ). The remaining levels did not show any significant differences regarding mean lung density, yet all the levels in PE group had lower mean lung densities (Table 2). Histogram plots were shown (Figure 3).

The cardiac rotation angle was measured as 46.71 $\pm 8.49^{\circ}$ and $35.82 \pm 5.38^{\circ}$ in the PE group and control group, respectively $(p<0.001)$. There was a significant correlation between the cardiac rotation angle and Haller index in the PE group $(\rho=0.593 ; p<0.001)$.

Table 1. Patients' characteristics, imaging, and clinical features

\begin{tabular}{|c|c|c|c|}
\hline Factor & Pectus excavatum & Control & $p$-value \\
\hline Age $^{*}$ & $14.42 \pm 2.25$ & $14.61 \pm 2.09$ & 0.73 \\
\hline Sex (female/male) & $9 / 24$ & $6 / 25$ & 1 \\
\hline Cardiac rotation angle* & $46.71 \pm 8.49$ & $35.82 \pm 5.38$ & $<0.001$ \\
\hline Haller index* & $3.53 \pm 0.66$ & $2.05 \pm 0.14$ & \\
\hline Female* & $3.39 \pm 0.98$ & $1.98 \pm 0.12$ & \\
\hline Male* & $3.56 \pm 0.56$ & $2.05 \pm 0.13$ & $<0.001$ \\
\hline \multicolumn{4}{|l|}{ Pulmonary function tests } \\
\hline $\mathrm{FEV}_{1}^{*}$ & $89.83 \pm 10.32$ & - & \\
\hline $\mathrm{FVC}^{*}$ & $83 \pm 10.90$ & - & \\
\hline $\mathrm{FEV}_{1} / \mathrm{FVC}^{*}$ & $105.26 \pm 6.66$ & - & \\
\hline
\end{tabular}

*Mean \pm standard deviations. FVC - forced vital capacity, FEV1 - forced expiratory volume in 15
There were no correlations between any levels of the mean lung density and cardiac rotation angle and Haller index (Table 3). The mean lung density of the T10R lung level for children with Haller index $<3.2$ was $815.84 \pm$ $38.92 \mathrm{HU}$ and for Haller index $>3.2$ it was $820.40 \pm 30.38$ $\mathrm{HU}$. The difference was not significant $(p=0.854)$.

No significant differences were found between the sex groups and cardiac rotation angle and Haller index measurements in the PE group $(p=0.378 ; p=0.725$, respectively).

Pulmonary function tests were available in all PE patients. The spirometry results were normal in 21 of 33 patients $(63.6 \%)$, obstructive pattern in 4 of 33 patients (12.1\%), and restrictive pattern in 8 of 33 patients (24.2\%). The mean values of the Haller index and the mean lung densities of each level did not show any significant difference between normal, obstructive, and restrictive pattern in pulmonary function tests (Table 4). No significant correlations were found between mean lung densities of each level and pulmonary function tests (Table 3).

\section{Discussion}

Lung parenchyma evaluation in patients with PE is important because PE causes not only cosmetic but also cardiopulmonary problems [2-5]. This is the first study evaluating the lung density of children with PE by a sensitive and objective method: quantitative CT. We demonstrated that all the lung levels had lower mean densities in the PE group compared to healthy children, with statistical significance in the right lung at the T10 vertebra level without any correlation with the Haller index, cardiac rotation angle, and pulmonary function tests. According to our results, we can speculate that the lower mean parenchymal densities may occur in children with PE regardless of the severity. Although most of the patients had normal pulmonary function test results, the lower mean lung densities on quantitative CT might indicate the initial phase of chronic lung changes.

Automated quantitative CT analysis provides more accurate results in the measurements of density, hetero-

Table 2. The comparison of the mean lung densities between the pectus excavatum and control groups

\begin{tabular}{|l|c|c|c|}
\hline Mean lung density & $\begin{array}{c}\text { Pectus excavatum } \\
\text { Mean } \pm \text { SD }\end{array}$ & $\begin{array}{c}\text { Control } \\
\text { Mean } \pm \text { SD }\end{array}$ & $p$-value \\
\hline T4R mean lung density & $-814.75 \pm 33.61$ & $-793.19 \pm 42.67$ & 0.050 \\
\hline T4L mean lung density & $-817.48 \pm 39.66$ & $-801.23 \pm 40.67$ & 0.094 \\
\hline T10R mean lung density & $-818.60 \pm 33.49$ & $-798.45 \pm 40.24$ & 0.028 \\
\hline T10L mean lung density & $-810.42 \pm 39.25$ & $-796.79 \pm 41.89$ & 0.202 \\
\hline CRAR mean lung density & $-813.03 \pm 40.02$ & $-797.06 \pm 36.33$ & 0.087 \\
\hline CRAL mean lung density & $-810.77 \pm 41.72$ & $-787.77 \pm 39.44$ & 0.060 \\
\hline
\end{tabular}

T4R - the right lung at the T4 vertebra spinous process level, T4L - the left lung at the T4 vertebra spinous process level, T10R - the right lung at the T10 vertebra spinous process level, $\mathrm{T} 10 \mathrm{~L}$ - the left lung at the T10 vertebra spinous process level, CRAR - the right lung at the level of the measurement of cardiac rotation angle, CRAL - the left lung at the level of the measurement of cardiac rotation angle 
A

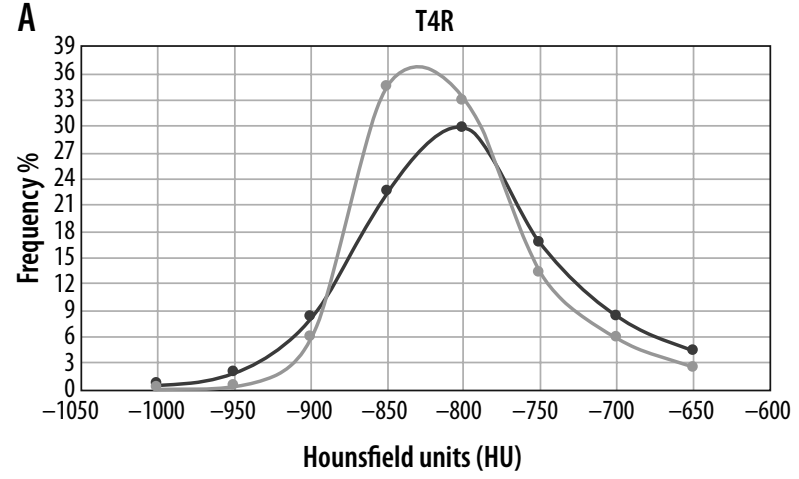

C

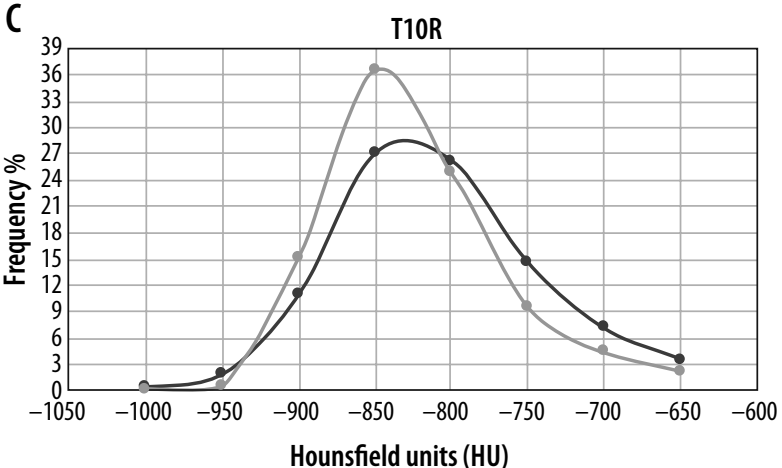

E

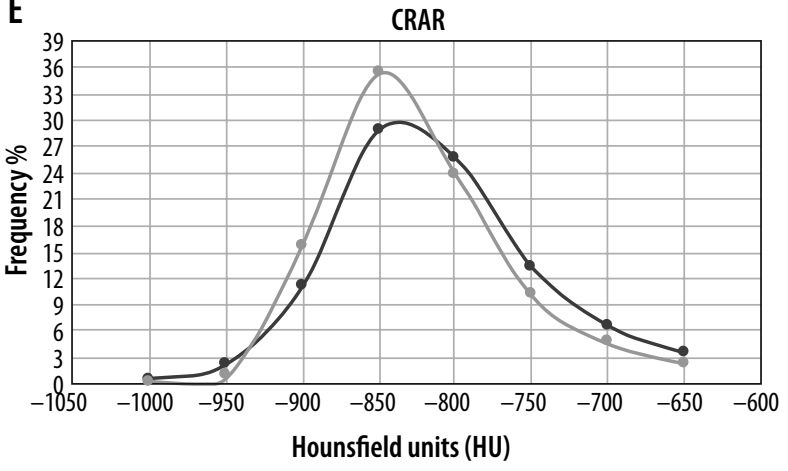

B

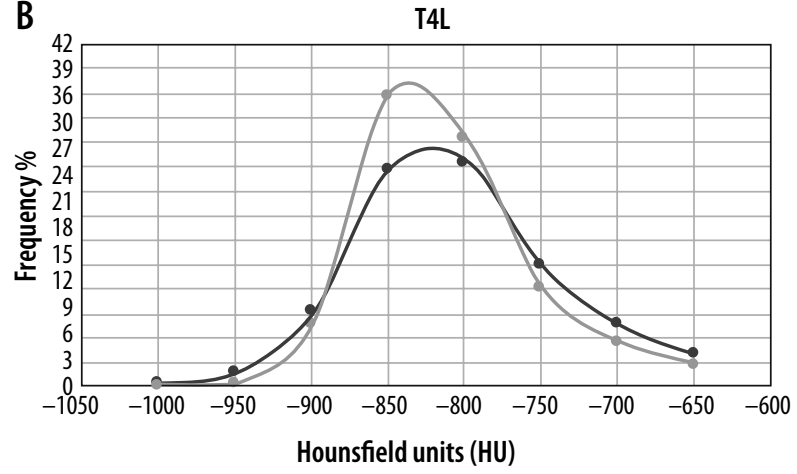

D

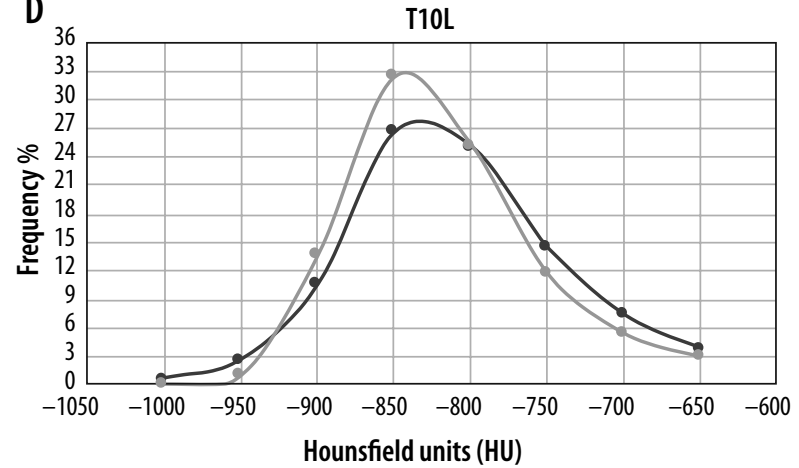

F

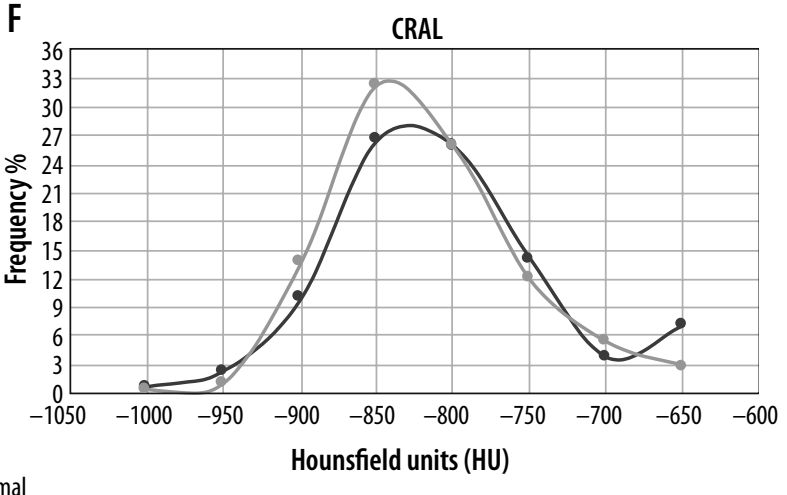

$\rightarrow$ - norme

Figure 3. Histogram plots of the lung levels. T4R - the right lung at the T4 vertebra spinous process level; $T 4 \mathrm{~L}$ - the left lung at the T4 vertebra spinous process level; T10R - the right lung at the T10 vertebra spinous process level; $\mathrm{T} 10 \mathrm{~L}$ - the left lung at the T10 vertebra spinous process level; (RAR - the right lung at the level of the measurement of cardiac rotation angle; CRAL - the left lung at the level of the measurement of cardiac rotation angle

geneity, tissue, vascular, and airway by eliminating the intra- and interobserver variability [8]. There have been many studies regarding utilities of automated densitometric analysis by quantitative CT in adults [9-12]. However, only a few studies have been published including the use of an automated quantitative densitometric CT analysis in the paediatric age group [17-19]. Our study is the first article to calculate the lung parenchyma density by automated quantitative $\mathrm{CT}$ in children with PE.

Chest CT allows determination of several thoracic indexes to assess the severity of the chest wall deformity and to guide surgical strategies [19]. Although the degree of chest wall deformity is estimated objectively by the Haller index and correction index, the Haller index is commonly used to establish surgical candidacy [19]. Normal mean values of the Haller index are described as less than 2.5 [13]. The cut-off value of greater than 3.25 is defined for severe
$\mathrm{PE}$, and it is used as a surgical indicator according to the guidelines $[14,15]$. It is known that the phase of respiration at the time of imaging affects the Haller index $[20,21]$. We used inspiratory phase images in children because the cooperation for breath-holding was easier in children.

The mediastinal shift, cardiac compression, and parenchymal abnormalities can also be determined by chest CT. There have been several articles that have assessed sternal depression, cardiac rotation [7], Haller index [22], and parenchymal abnormalities $[5,23]$ by using $\mathrm{CT}$ in patients with PE. In our study, the cardiac rotation angle increment was correlated with the Haller index in children with PE. The sternal depression may cause a right ventricular compression, and accordingly cardiovascular impairment may occur [24]. Chu et al. [7] reported that there was a positive correlation between the sternal depression index and the degree of cardiac rotation. 
Table 3. The correlation coefficients between mean lung densities and pulmonary functions tests, cardiac rotation angle, and Haller index

\begin{tabular}{|l|c|c|c|c|c|c|c|c|c|c|}
\multirow{2}{*}{ Mean lung density } & \multicolumn{2}{|c|}{ FEV $_{1}$} & \multicolumn{2}{c|}{ FVC } & \multicolumn{3}{c|}{ FEV /FVC $_{1}$} & \multicolumn{2}{c|}{$\begin{array}{c}\text { Cardiac rotation } \\
\text { angle }\end{array}$} & \multicolumn{2}{c|}{ Haller index } \\
\cline { 2 - 12 } & $\rho$ & $p$-value & $\rho$ & $p$-value & $\rho$ & $p$-value & $\rho$ & $p$-value & $\rho$ & $p$-value \\
\hline T4R mean lung density & -0.192 & 0.379 & -0.016 & 0.943 & -0.048 & 0.829 & 0.168 & 0.349 & 0.130 & 0.471 \\
\hline T4L mean lung density & -0.520 & 0.488 & 0.021 & 0.973 & -0.021 & 0.923 & 0.240 & 0.179 & 0.194 & 0.281 \\
\hline T10R mean lung density & -0.186 & 0.394 & -0.020 & 0.961 & 0.020 & 0.928 & 0.250 & 0.160 & 0.203 & 0.258 \\
\hline T10L mean lung density & -0.134 & 0.543 & -0.129 & 0.796 & 0.129 & 0.556 & 0.327 & 0.063 & 0.153 & 0.394 \\
\hline CRAR mean lung density & -0.166 & 0.450 & -0.077 & 0.753 & 0.077 & 0.728 & 0.248 & 0.165 & 0.233 & 0.192 \\
\hline CRAL mean lung density & -0.122 & 0.580 & 0.019 & 0.995 & 0.019 & 0.932 & 0.247 & 0.165 & 0.198 & 0.271 \\
\hline
\end{tabular}

T4R - the right lung at the T4 vertebra spinous process level, T4L - the left lung at the T4 vertebra spinous process level, T10R - the right lung at the T10 vertebra spinous process level, $\mathrm{T} 10 \mathrm{~L}$ - the left lung at the T10 vertebra spinous process level, CRAR - the right lung at the level of the measurement of cardiac rotation angle, CRAL - the left lung at the level of the measurement of cardiac rotation angle, FVC - forced vital capacity, $\mathrm{FEV}_{1}$ - forced expiratory volume in $1 \mathrm{~s}$

Table 4. The mean lung density of each lung levels and Haller index in PE patients according to lung function

\begin{tabular}{|l|c|c|c|c|}
\hline Mean lung density & $\begin{array}{c}\text { Normal lung function } \\
(n=21) \\
\text { Mean } \pm \text { SD }\end{array}$ & $\begin{array}{c}\text { Obstructive lung function } \\
(n=4) \\
\text { Mean } \pm \text { SD }\end{array}$ & $\begin{array}{c}\text { Restrictive lung function } \\
(n=8) \\
\text { Mean } \pm \text { SD }\end{array}$ & $p$-value* \\
\hline T4R mean lung density & $-817.49 \pm 44.27$ & $-819.31 \pm 24.16$ & $-830.52 \pm 23.22$ & 0.893 \\
\hline T4L mean lung density & $-818.95 \pm 52.30$ & $-821.69 \pm 27.63$ & $-835.52 \pm 23.83$ & 0.788 \\
\hline T10R mean lung density & $-820.92 \pm 43.75$ & $-828.39 \pm 20.07$ & $-821.34 \pm 18.33$ & 0.994 \\
\hline T10L mean lung density & $-814.32 \pm 52.22$ & $-810.17 \pm 32.45$ & $-827.24 \pm 16.08$ & 0.865 \\
\hline CRAR mean lung density & $-815.46 \pm 50.05$ & $-810.33 \pm 30.55$ & $-828.20 \pm 28.94$ & 0.731 \\
\hline CRAL mean lung density & $-811.47 \pm 54.60$ & $-827.86 \pm 22.46$ & $-827.32 \pm 22.28$ & 0.966 \\
\hline Haller index & $3.49 \pm 0.66$ & $3.90 \pm 0.56$ & $3.51 \pm 0.50$ & 0.487 \\
\hline
\end{tabular}

*Kruskal-Wallis test was used. T4R - the right lung at the T4 vertebra spinous process level, T4L - the left lung at the T4 vertebra spinous process level, T10R - the right lung at the T10 vertebra spinous process level, T10L - the left lung at the T10 vertebra spinous process level, CRAR - the right lung at the level of the measurement of cardiac rotation angle, CRAL - the left lung at the level of the measurement of cardiac rotation angle

Singh et al. [5] proposed that both restriction of thorax anterior-posterior diameter and displacement of the heart to the left were the reasons for the compression of the bronchus and subsequently air being trapped in the lower lobe anterior basal segment. Our results did not support this theory. We suggest that lower mean lung densities might be associated with increased aeration, which may occur as a compensatory response to relatively pressed lungs due to the lower thoracic volume from birth. Also, the increased aeration in both sides of the upper and lower lobes may be explained by the fact that the disease is congenital.

Hyperinflation of the left lower lobe anterior basal segment was reported in patients with PE in a study conducted by Singh et al. [5] They also noted that this finding showed an association with the PE severity. They evaluated the presence or absence of left lower lobe hyperinflation by visual inspection and drawing manually a $1 \mathrm{~cm}^{2}$ diameter ROI in the left and right anterior basal segments. It is known that the ROI provides information about the local density only in the selected area. As expected, focal hyperinflation could be observed by a small ROI that was placed on the visually selected focal parenchymal hypodense area. In our study, lower values of the mean parenchymal densities were noticed at all the levels, not at a focal area, in the PE group, with a statistical significance in the right lower lobe. Although our study contained a relatively small number of cases, we thought that we obtained more accurate results due to the sensitive nature of our measurement technique. The reason for not finding statistically significant lower results at all the levels in patients with PE may be the small study population. Additionally, the non-correlation between the mean lung densities and the Haller index in this study showed that the parenchymal hyperinflation develops independently of the severity of PE.

There are inconsistent results in the literature regarding whether the thoracic wall deformity in the PE causes an effect on pulmonary function [3-5,25-28]. Despite these controversies, pulmonary function tests are used as a surgical indicator and are performed for surgical candidates [15]. Most of the patients had normal spirometry results in our study (65.2\%). Additionally, no significant relationships were found between the pulmonary function tests and mean lung densities in each level of the lung, as well as the Haller index severity and cardiac rotation angle. These 
results may suggest that pulmonary function tests should be performed regardless of the severity of the Haller index and cardiac rotation in patients with PE. Our results were compatible with a study of Singh et al. [5]. Restrictive lung disease was the second most common spirometry finding in our study (21.7\%). In a study by Lawson et al. [3] with a large cohort, it was reported that the restrictive pattern was more prominent than the obstructive pattern in $\mathrm{PE}$ patients. Additionally, the lung restriction was significantly related to the severity of the chest wall deformity. Non-correlated results between the pulmonary function tests and the Haller index in our study may be associated with the small number of patients in the group.

Our study has some limitations. First, we examined a relatively small sample size. Although many patients' images had enough quality for preoperative evaluation, all the patients who had minor artifacts were excluded for reliable and accurate densitometric analysis. Second, the lung density and the Haller index are related to the phase of breathing on chest CT scans. Inspiratory images alone are unlikely to be the best option to check the effect of PE on chest mechanics. However, as in our hospital and in many centres, chest CT scans are done with a single phase routinely in children to minimize the effects of the radiation.

\section{Conclusions}

This study demonstrated lower mean lung densities in children with PE compared to healthy children, especially in the right lower lobe. However, the lower mean lung densities were not associated with either the Haller index or cardiac rotation. Pulmonary function tests were also not correlated with mean lung densities. We recommend considering the parenchymal aeration independently from the severity of PE. Finally, further prospective research regarding the potential clinical significance and long-term assessment is needed because there may be cardiopulmonary consequences of the decreased mean lung density.

\section{Conflict of interest}

The authors report no conflict of interest.

\section{References}

1. Chung CS, Myrianthopoulos NC. Factors affecting risks of congenital malformations. I. Analysis of epidemiologic factors in congenital malformations. Report from the collaborative perinatal project. Birth Defects Orig Artic Ser 1975; 11: 1-22.

2. Morshuis WJ, Mulder H, Wapperom G, et al. Pectus excavatum. A clinical study with long-term postoperative follow-up. Eur J Cardiothorac Surg 1992; 6: 318-328.

3. Lawson ML, Mellins RB, Paulson JF, et al. Increasing severity of pectus excavatum is associated with reduced pulmonary function. J Pediatr 2011; 159: 256-261.

4. Fonkalsrud EW, Salman T, Guo W, Gregg JP. Repair of pectus deformities with sternal support. J Thorac Cardiovasc Surg 1994; 107: 37-42.

5. Singh S, Greenberg SB. Hyperinflation of the left anterior basal segment: a new sign of pectus excavatum severity on CT. Clin Radiol 2017; 72: 991.e15-991.e18.

6. Tocchioni F, Ghionzoli M, Messineo A, Romagnoli P. Pectus excavatum and heritable disorders of the connective tissue. Pediatr Rep 2013; 5: e15.

7. Chu ZG, Yu JQ, Yang ZG, Peng LQ, Bai HL, Li XM. Correlation between sternal depression and cardiac rotation in pectus excavatum: evaluation with helical CT. AJR Am J Roentgenol 2010; 195: 76-80.

8. Herth FJF, Kirby M, Sieren J, et al. The modern art of reading computed tomography images of the lungs: quantitative CT. Respiration 2018; 95: 8-17.

9. Nambu A, Zach J, Schroeder J, et al. Quantitative computed tomography measurements to evaluate airway disease in chronic obstructive pulmonary disease: relationship to physiological measurements, clinical index and visual assessment of airway disease. Eur J Radiol 2016; 85: 2144-2151.
10. Obert M, Kampschulte M, Limburg R, et al. Quantitative computed tomography applied to interstitial lung diseases. Eur J Radiol 2018; 100: 99-107.

11. Kumar I, Verma A, Jain A, Agarwal SK. Performance of quantitative CT parameters in assessment of disease severity in COPD: a prospective study. Indian J Radiol Imaging 2018; 28: 99-106.

12. Witt CA, Sheshadri A, Carlstrom L, Agarwal SK. Longitudinal changes in airway remodeling and air trapping in severe asthma. Acad Radiol 2014; 21: 986-993.

13. Haller JA, Jr, Kramer SS, Lietman SA. Use of CT scans in selection of patients for pectus excavatum surgery: a preliminary report. J Pediatr Surg 1987; 22: 904-906.

14. Sidden CR, Katz ME, Swoveland BC, Nuss D. Radiologic considerations in patients undergoing the Nuss procedure for correction of pectus excavatum. Pediatr Radiol 2001; 3: 429-434.

15. Nuss D, Obermeyer RJ, Kelly RE. Nuss bar procedure: past, present and future. Ann Cardiothorac Surg 2016; 5: 422-433.

16. Miller MR, Hankinson J, Brusasco V, et al. Standardisation of spirometry. Eur Respir J 2005; 26: 319-338.

17. Stein JM, Walkup LL, Brody AS, Woods JC. Quantitative CT characterization of pediatric lung development using routine clinical imaging. Pediatr Radiol 2016; 46: 1804-1812.

18. Kim J, Kim MJ, Sol IS, Fleck RJ, Wood JC. Quantitative CT and pulmonary function in children with post-infectious bronchiolitis obliterans. PLoS One 2019; 14: e0214647.

19. Rodríguez-Granillo GA, Martínez-Ferro M, Capuñay C, et al. Preoperative multimodality imaging of pectus excavatum: state of the art review and call for standardization. Eur J Radiol 2019; 117: 140-148. 20. Albertal M, Vallejos J, Bellia G, et al. Changes in chest compression indexes with breathing underestimate surgical candidacy in patients 
with pectus excavatum: a computed tomography pilot study. J Pediatr Surg 2013; 48: 2011-2016.

21. Birkemeier KL, Podberesky DJ, Salisbury S, Serai S. Breathe in... breathe out... stop breathing: does phase of respiration affect the Haller index in patients with pectus excavatum? AJR Am J Roentgenol 2011; 197: W934-W939.

22. Daunt SW, Cohen JH, Miller SF. Age-related normal ranges for the Haller index in children. Pediatr Radiol 2004; 34: 326-330.

23. Wang H, Wang FH, Liang JH, et al. Pectus excavatum and congenital cystic lung lesion: simultaneous surgery advocated. J Thorac Dis 2018; 10: 6230-6237.

24. Park SY, Park TH, Kim JH, et al. A case of right ventricular dysfunction caused by pectus excavatum. J Cardiovasc Ultrasound 2010; 18: 62-65.
25. Kelly RE Jr, Obermeyer RJ, Nuss D. Diminished pulmonary function in pectus excavatum: from denying the problem to finding the mechanism. Ann Cardiothorac Surg 2016; 5: 466-475.

26. Malek MH, Berger DE, Marelich WD, Coburn JW, Beck TW, Housh TJ. Pulmonary function following surgical repair of pectus excavatum: a meta-analysis. Eur J Cardiothorac Surg 2006; 30: 637643.

27. Sesia SB, Obermeyer RJ, Mayr J, Haecker FM. Pulmonary function in pectus excavatum patients before repair with the Nuss procedure. Postgrad Med 2016; 128: 598-602.

28. Neviere R, Montaigne D, Lotfi B, et al. Cardiopulmonary response following surgical repair of pectus excavatum in adult patients. Eur J Cardiothorac Surg 2011; 40: e77-e82. 